\title{
Pre-Kidney Transplant Screening for Coronary Artery Disease: Current Practice in the United Kingdom
}

\author{
Ailish Nimmo ${ }^{1 *}$, Matthew Graham-Brown ${ }^{2,3}$, Sian Griffin $^{4}$, Adnan Sharif ${ }^{5}$, Rommel Ravanan ${ }^{1}$ \\ and Dominic Taylor ${ }^{1}$ \\ ${ }^{1}$ North Bristol NHS Trust, Bristol, United Kingdom, ${ }^{2}$ University of Leicester, Leicester, United Kingdom, ${ }^{3}$ University Hospitals of \\ Leicester NHS Trust, Leicester, United Kingdom, ${ }^{4}$ Cardiff and Vale University Health Board, Cardiff, United Kingdom, ${ }^{5}$ Queen \\ Elizabeth Hospital Birmingham, Birmingham, United Kingdom
}

Keywords: kidney transplantation, screening, cardiovascular disease, survey, risk factors

Dear Editors,

Randomised control trial (RCT) evidence is not available to guide screening for asymptomatic coronary artery disease before kidney transplantation [1]. United Kingdom observational data show no clear benefit from screening [2]. To gain data representative of current practice in the United Kingdom, we invited a lead transplant nephrologist from each kidney transplant centre to complete a survey examining cardiac screening practice, work-up pathways, and appetite for a national RCT in June 2021. Ethical approval was not required.

Responses were received from all $23(100 \%)$ centres, of which 22 had a protocol for cardiac assessment prior to listing. In three centres, asymptomatic individuals were not required to undergo cardiac investigation beyond an ECG or echocardiogram prior to transplantation. The remainder followed a risk-stratified approach; no centres performed universal screening.

In centres adopting risk-stratified screening, factors used to screen patients included a history of ischaemic heart disease (100\% of centres), diabetes (100\%), peripheral vascular disease $(50 \%)$, smoking (50\%), stroke (35\%), limited exercise capacity (35\%), hyper/hypotension (15\%), or an abnormal echocardiogram (95\%) or ECG (70\%). Two centres stratified using the Newcastle Risk Index [3]. Thirteen centres had a specific age threshold (mostly 50 or 60 years), whilst others included age in combination with additional risk factors or Newcastle Risk Index scores.

The most frequent screening investigation was a myocardial perfusion scan (55\%) followed by stress echocardiogram (20\%). Coronary angiography and cardiopulmonary exercise testing were the initial investigation in one centre each. Other indications for coronary angiography included an abnormal initial screening test (39\%) or on cardiology advice (35\%). In one third of centres, the waiting time for investigations was over 10 weeks.

Nine centres had cardio-renal multidisciplinary meetings, whilst 14 had a designated cardiologist providing transplant candidate assessments. In 16 centres cardiology review was only needed for patients with abnormal screening tests, whilst in three cardiologists reviewed all screened patients.

Of 23 centres, 10 had updated their screening protocol within the past 2 years and three were in the

Received: 17 September 2021 Accepted: 29 November 2021

Published: 07 January 2022

Citation:

Nimmo A, Graham-Brown M, Griffin S,

Sharif $A$, Ravanan $R$ and Taylor $D$

(2022) Pre-Kidney Transplant

Screening for Coronary Artery Disease:

Current Practice in

the United Kingdom.

Transpl Int 35:10039.

doi: 10.3389/ti.2021.10039

Abbreviations: ECG, Electrocardiogram; RCT, Randomised controlled trial. 


\section{Pre-transplant cardiac screening: practice in all 23 UK kidney transplant centres}

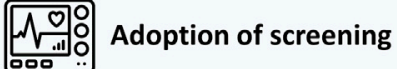

No screening beyond ECG/echo: $13 \%$

Risk-stratified screening: $87 \%$

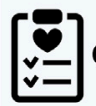
Criteria in risk-stratified protocols

Diabetes: $100 \%$

Ischaemic heart disease: $100 \%$

Age: $95 \%$ ( 50 years $32 \%, 60$ years $39 \%$ )

Abnormal echo: $95 \%$

Abnormal ECG: $70 \%$

Peripheral vascular disease: $50 \%$

Smoking: $50 \%$

Stroke: $35 \%$

Limited exercise capacity: $35 \%$

Hyper/hypotension: $15 \%$

Newcastle Risk Index Score: 10\%
Initial investigation

Myocardial perfusion scan: 55\%

Stress echo: $20 \%$

Exercise tolerance test: 15\%

Cardiopulmonary exercise test: $5 \%$

Coronary angiogram: $5 \%$

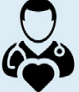

Cardiology involvement

Cardio-renal multidisciplinary meeting: 39\%

Named transplant cardiologist: $61 \%$

Cardiology review if abnormal screening test: $70 \%$

Cardiology review of all patients needing a screening test: $13 \%$
Involved in listing decisions

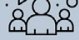

Transplant surgeon: $100 \%$

Nephrologist: 100\%

Anaesthetist: $56 \%$

Cardiologist: 39\%

\section{Abnormal screening tests}

19 centres had experience of not listing patients based solely on screening test

$<1$ patient per month: $58 \%$

1-5 patients per month: $21 \%$

Unsure: $21 \%$

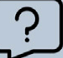

Willing to take part in clinical trial

Yes: $96 \%$

FIGURE 1 | Summary of findings from survey of pre-transplant cardiac screening.

screening. Of 23 centres, 22 expressed interest to participate in an RCT to examine the utility of screening, 12 of whom supported recruiting the highest cardiac risk candidates.

Our survey highlights variation in screening practice across the United Kingdom (Figure 1). Similar heterogenous practice has been shown in the United States [4], although our survey was undertaken following publication of ISCHEMIA-CKD [5]. Whilst no centres perform universal screening and many have recently updated their protocols, which may represent a trend away from routine screening, responses highlight nephrologists' concerns over the evidence upon which practice is based. Capturing views of other transplant professionals and patients is essential, but this survey suggests support for an RCT to evidence utility of screening.

\section{DATA AVAILABILITY STATEMENT}

The raw data supporting the conclusions of this article will be made available by the authors, without undue reservation.

\section{ETHICS STATEMENT}

Ethical review and approval was not required for the study on human participants in accordance with the local legislation and institutional requirements. Written informed consent for participation was not required for this study in accordance with the national legislation and the institutional requirements.

\section{AUTHOR CONTRIBUTIONS}

All authors contributed to study design and writing of the survey questions. AN performed the analyses, produced the figure and wrote the letter under the supervision of RR and DT. All authors contributed to manuscript preparation.

\section{CONFLICT OF INTEREST}

The authors declare that the research was conducted in the absence of any commercial or financial relationships that could be construed as a potential conflict of interest.

\section{ACKNOWLEDGMENTS}

We would like to thank the respondents to this survey. 


\section{REFERENCES}

1. Sharif A. The Argument for Abolishing Cardiac Screening of Asymptomatic Kidney Transplant Candidates. Am J Kidney Dis (2020). 75(6):946-54. doi:10.1053/j.ajkd.2019.05.033

2. Nimmo A, Forsyth JL, Oniscu GC, Robb M, Watson C, Fotheringham J, et al. A Propensity Score-Matched Analysis Indicates Screening for Asymptomatic Coronary Artery Disease Does Not Predict Cardiac Events in Kidney Transplant Recipients. Kidney Int (2021). 99(2):431-42. doi:10.1016/ j.kint.2020.10.019

3. Asher J, Oliver A, Wilson C, Gupta A, Gok M, Balupuri S, et al. A Simple Cardiovascular Risk Score Can Predict Poor Outcome in NHBD Renal Transplantation. Transplant Proc (2005). 37(8):3292-3. doi:10.1016/ j.transproceed.2005.09.136
4. Cheng XS, Mathew RO, Parasuraman R, Tantisattamo E, Levea S-L, Kapoor R, et al. Coronary Artery Disease Screening of Asymptomatic Kidney Transplant Candidates: A Web-Based Survey of Practice Patterns in the United States. Kidney Med (2020). 2(4):505-7. doi:10.1016/j.xkme.2020.04.006

5. Bangalore S, Maron DJ, O'Brien SM, Fleg JL, Kretov EI, Briguori C, et al. Management of Coronary Disease in Patients with Advanced Kidney Disease. N Engl J Med (2020). 382(17):1608-18. doi:10.1056/NEJMoa1915925

Copyright (c) 2022 Nimmo, Graham-Brown, Griffin, Sharif, Ravanan and Taylor. This is an open-access article distributed under the terms of the Creative Commons Attribution License (CC BY). The use, distribution or reproduction in other forums is permitted, provided the original author(s) and the copyright owner(s) are credited and that the original publication in this journal is cited, in accordance with accepted academic practice. No use, distribution or reproduction is permitted which does not comply with these terms. 